\title{
Application of NAA to air particulate matter collected at thirteen sampling sites in eight Asian countries: A collaborative study
}

\author{
M. Ebihara, ${ }^{1}{ }^{*}$ Y. S. Chung, ${ }^{2}$ H. M. Dung, ${ }^{3}$ J. H. Moon, ${ }^{2}$ B.-F. Ni, ${ }^{4}$ T. Otoshi, ${ }^{5}$ Y. Oura, ${ }^{1}$ F. L. Santos, ${ }^{6}$ \\ F. Sasajima, ${ }^{7}$ Sutisna, ${ }^{8}$ B. S. Wee, ${ }^{1,9}$ W. Wimolwattanapun, ${ }^{10}$ A. K. B. H. Wood ${ }^{9}$ \\ ${ }^{I}$ Department of Chemistry, Tokyo Metropolitan University, Hachioji, Tokyo 192-0397, Japan \\ ${ }^{2}$ Korea Atomic Energy Research Institute, Daejeon, Korea \\ ${ }^{3}$ Nuclear Research Institute, Dalat, Vietnam \\ ${ }^{4}$ China Institute of Atomic Energy, Beijing, P.R. China \\ ${ }^{5}$ Tohoku University of Community Services and Science, Sakata, Japan \\ ${ }^{6}$ Philippine Nuclear Research Institute, Quezon City, Philippines \\ ${ }^{7}$ Japan Atomic Energy Research Institute, Tokai, Japan \\ ${ }^{8}$ National Nuclear Energy Agency, Serpong, Indonesia \\ ${ }^{9}$ Malaysian Nuclear Agency, Bangi, Selangor, Malaysia \\ ${ }^{10}$ Thailand Institute of Nuclear Technology, Bangkok, Thailand
}

(Received July 10, 2008)

\begin{abstract}
Air particulate matter (APM) samples $\left(\mathrm{PM}_{2.5}\right.$ and $\left.\mathrm{PM}_{10-2.5}\right)$ were collected at 13 sampling points in 8 Asian countries and their chemical compositions were determined by using neutron activation analysis (NAA) with the $k_{0}$-standardization method in addition to conventional comparative method of NAA. Analytical data showed that mass concentration and elemental composition of the APM collected are variable in terms of time and space, and are related to the characteristics of the sampling sites concerned. NAA was proved to be highly effective for the regional characterization of APM in chemical composition.
\end{abstract}

\section{Introduction}

A collaborative work was initiated through the Forum for Nuclear Cooperation in Asia (FNCA) sponsored by the Japanese Government in 2000, to which China, Indonesia, Japan, Korea, Malaysia, Philippines, Thailand and Vietnam are allied to work together under a general theme of the utilization of research reactor. With this theme, an application of neutron activation analysis (NAA) was adopted as a subtheme in recognition of its importance amongst the participating countries. An emphasis of this collaborative study has been placed on the utilization of NAA in analyzing environmental samples. NAA is capable of determining about 30 elements in air particulate matter (APM) by applying appropriate irradiation, cooling and counting procedures. ${ }^{1}$ With more proper optimization of these procedures, 42 elements could be detected. ${ }^{2}$ Thus, NAA has been proved to be effective in getting reliable data for APM.

This paper aims to focus on the analysis of APM collected at 13 sampling points in 8 Asian countries in the years 2003 and 2004, and to characterize mass and elemental concentrations of APM in terms of several parameters involved in sampling of APM. The APM analyzed consists of fine $\left(\mathrm{PM}_{2.5}\right)$ and coarse $\left(\mathrm{PM}_{10-2.5}\right)$ fractions. Both temporal and spatial variations of mass concentration and chemical composition are discussed. This paper is an extension of our previous report. ${ }^{1}$

\section{Experimental}

\section{Sampling protocol}

Sampling sites for this study were carefully chosen to include urban, industrial and rural sites. Descriptions of the sampling sites are given in our previous report ${ }^{1}$ except for those in Vietnam, where APM was collected at two sampling sites of Ho Chi Minh (industrial site) and Dateh (rural area). APM was collected by using a Gent air sampler or an equivalent installed with two polycarbonate filters having pore sizes of 0.4 and $8 \mu \mathrm{m}$. APM was thus separately sampled into fine and coarse fractions, which are referred to $\mathrm{PM}_{2.5}$ and $\mathrm{PM}_{10-2.5}$ in this paper. The sampling time varied from 24 hours to a few days with a sampling rate of $16.7 \mathrm{~L} / \mathrm{min}$.

\section{Sample analysis}

NAA using reactor neutrons was utilized for the analysis of APM. NIST 1632c (Bituminous Coal) and NIES No. 8 (Vehicle Exhaust Particulates) were also analyzed as quality control. ${ }^{1}$ Both short and long irradiations were performed in order to determine various radionuclides with different half-lives. For short irradiation, air filters were irradiated for about 2-10 minutes with subsequent cooling of 2-30 minutes. For long irradiation, air filters were irradiated for 2-16 hours, with cooling time ranging from 1 to 30 days. With appropriate cooling, samples were counted for 